\title{
Position and Processing of Adverse Drug Reactions Directly Submitted by Patients to National Regulatory Authorities in Europe
}

\author{
Jindrich Srba* and Jiri Vlcek
}

Department of Social and Clinical Pharmacy, Faculty of Pharmacy, Charles University, Hradec Kralove, Czech Republic

\begin{abstract}
Introduction: Adverse drug reaction (ADR) reports submitted by patients to national regulatory authorities should be directly accepted since July 2012 when new pharmacovigilance legislation was implemented. This questionnairebased analysis and review was conducted to evaluate the position of ADR reports submitted by patients and to compare ADR reporting by patients and healthcare professionals (HPs).

Materials and Methods: Questionnaires were provided to national regulatory authorities in 2011 to evaluate their attitudes toward the ADR reports submitted directly by patients. In addition, electronic databases (2003-2011) were searched, including MEDLINE and EMBASE, and reference lists of included studies were reviewed, to compare data from ADR reports sent by patients and HPs.

Results: Of the 30 questionnaires distributed to national regulatory authorities, 17 were received back and included in this analysis. Patients were allowed to directly submit ADR reports in 12 countries. ADR reports from patients were used for signal detection in 10 countries. Medical confirmation was performed in four countries. Four comparative studies were included in systematic review. Varying outcomes were observed across the studies based on qualitative analysis.

Conclusions: Different approach in the processing of ADR reports submitted by patients among national regulatory authorities was observed. Comparison of reports submitted by patients and HPs resulted in various outcomes. Position of patient reporting was not uniform and varies from country to country. Further investigation of the processing of ADR reports would be beneficial to better understand the potential or reports submitted by patients.
\end{abstract}

Keywords: Adverse Drug Reaction; Reporting; Pharmacovigilance; Patient; National Regulatory Authority

\section{Introduction}

National pharmacovigilance systems in Europe were established in the 1960s [1]. Pharmacovigilance activities are maintained by national regulatory authorities responsible for collecting adverse drug reaction (ADR) reports from healthcare professionals (HPs), marketing authorization holders, patients, and other sources [2].

Since July 2012, ADR report directly submitted by patient to national regulatory authority should be always accepted based on the new European legislation Regulation (EU) No 1235/2010 and Directive 2010/84/EU [3,4]. The European Commission reviewed the system and proposed new EU pharmacovigilance legislation, in order to continue to improve patient safety. The legislation was the biggest change to the regulation of human medicines in the European Union since 1995. One of the key aspects of implementation the new European legislation is to take account of, and encourage, the growing involvement of patients in the reporting of ADRs. In fact, this process began since 2003. ADR reports directly submitted by patients started in Denmark and the Netherlands since 2003, in the United Kingdom since 2005, in Sweden since 2008, or in Norway since 2010 [5-7]. On the other hand, there were countries not actively collecting patient reports like Finland, France, Germany, Ireland, Portugal or Spain, as it was investigated by Herxheimer et al. [8].

Decision to include patients as additional source of directly submitted ADR reports was based on a few articles concerned with this topic. An investigation by van den Bemt et al. confirmed HPs as the main source for reports of serious and unknown ADRs in hospitalized patients, yet patients seemed to report more ADRs to new drugs [9]. Following study by Jarernsiripornkul et al. suggested that patients do not report all of the symptoms that they suspect to be ADRs to their general practitioner and that the general practitioners do not record all of the symptoms [10]. This practice contributes to the underreporting, which is considered the main weakness of the current system [11]. Hence, patients as additional sources of reports are increasingly perceived to be important contributors to the spontaneous reporting system [12].

The main objective of this article was to characterize the position ADR reports directly submitted by patients in European countries before the introduction of the obligation to accept these reports from July 2012.

\section{Materials and Methods}

\section{Questionnaire-based analysis}

Analysis was done by survey using a self-administered structured questionnaire. Questionnaires were distributed on February 2011 to the general e-mail address of national regulatory authorities established in 30 different European countries (EEA member countries in 2011). Addressed subjects were asked for their response on March 2011. To increase the response rate, a second encouragement was conducted at

*Corresponding author: Jindrich Srba, Okrajová Department of Social and Clinical Pharmacy, Faculty of Pharmacy, Charles University, Hradec Kralove, Czech Republic Tel: +420 728645 080; Fax: +420 495518 002; E-mail: jindrich.srba@gmail.com

Received October 16, 2013; Accepted February 17, 2014; Published February 25,2014

Citation: Srba J, Vlcek J (2014) Position and Processing of Adverse Drug Reactions Directly Submitted by Patients to National Regulatory Authorities in Europe. J Pharmacovigilance 2: 122. doi:10.4172/2329-6887.1000122

Copyright: @ 2014 Srba J, et al. This is an open-access article distributed under the terms of the Creative Commons Attribution License, which permits unrestricted use, distribution, and reproduction in any medium, provided the original author and source are credited. 
the end of March 2011. In case of no response, email address of related pharmacovigilance department at national regulatory authority was used at the beginning of April 2011. Final questionnaire collection was concluded in April 2011.

The questionnaire consisted of two parts, qualitative and quantitative. For the first part, questions were related to the processing of ADR reports from patients. For the second part, the total numbers of ADR reports from patients in comparison with those from healthcare professionals from 2007 until 2010 were requested. The content of questionnaire was validated by the employee of pharmacovigilance department at State Institute for Drug Control in the Czech Republic. Validation was based on the evaluation of the relevance of the requested data and their availability at national regulatory authorities. Questionnaires were prepared in English language.

\section{Literature search}

A review was conducted in compliance with the PRISMA statement $[13,14]$. Studies related to the comparison of ADR reports submitted by patients and HPs were considered for inclusion. The search strategy was based on the electronic databases MEDLINE (Ovid) and EMBASE (Ovid). The search terms included: patients, consumers, healthcare professionals, physicians, adverse drug reactions, report, reporting, spontaneous, pharmacovigilance and surveillance. Text search terms and controlled vocabulary search terms for MEDLINE (MeSH) and EMBASE (EMTREE) were used. The searches covered the period from 1 Jan 2003 to 31 Dec 2011, inclusive. The search itself was performed on 23 May 2012. The decision to include an article was made primarily based on the title and abstract. Duplicate articles were detected and removed manually. In case of doubt, the full article was obtained for the final classification decision. Full-text articles were obtained for all of the selected articles.

Inclusion criteria: Articles published from 2003 to 2011 were included with the subject of the establishment of accepting ADR reports submitted by patients directly to national regulatory authorities. Articles published prior to 2003 were not included, because at that time any of the European countries actively supported the collection of ADR submitted by patients. To meet the inclusion criteria, the articles had to be prospective or retrospective studies, which investigated ADR reports submitted by HPs and patients to national regulatory authorities established in the European member states. Detected articles should compare one of the following criteria: reporter age and gender, most frequently reported ADRs and/or drugs, and the seriousness of the ADRs.

Exclusion criteria: Articles based solely on the analysis of reports from HPs or patients were excluded, to achieve the most uniform data dedicated to the comparison of reports from both sources. Reviews or meta-analyses were not included, as the main objective was to provide an overview of the studies concerned with the direct comparison of $\mathrm{ADR}$ reports from HPs and patients. Case reports and case series were also excluded.

Citation searching: The reference list of each included study was checked to identify further relevant research studies. The full paper was obtained for each study being considered for inclusion in this review.

\section{Results}

\section{Processing of ADR reports}

17 out of 30 national regulatory authorities sent back the questionnaires (response rate 57\%). In 12 countries, patients were allowed to report suspected ADRs by letter, telephone or via the internet. In 10 countries, ADR reports submitted directly by patients did not need to be medically confirmed and were directly accepted. Also in 10 countries, ADR reports submitted directly by patients were used for signal detection. In 6 countries, reports were directly accepted and used for signal detection at the same time: Denmark, Ireland, the Netherlands, Norway, Sweden and the United Kingdom (Table 1).

\section{Reporting ratio}

Reporting ratio describes the ratio of ADR reports from patients to the total number of ADR reports submitted to national regulatory authorities. Considerable variation of the reporting ratio exists across national systems per year (Figure 1). This variation was observed not only across different European countries, but also on a yearby-year basis. The contribution of ADR reports from patients to the total number of reports was 3\% in Ireland, 7\% in Norway, $8 \%$ in the United Kingdom, $16 \%$ in Sweden, $17 \%$ in the Netherlands, and $24 \%$ in Denmark during the period 2007-2010.

\section{Literature review}

A literature search produced a limited number of abstracts, very few of which were relevant. 4 articles included the assessment of ADR reports from patients and HPs in those European countries where ADR reports directly submitted by patients have been accepted. Two were from the Netherlands, one was from Denmark and one was from the United Kingdom (Figure 2).

\section{General findings}

The summary of the comparison of characteristics of the reporters, ADRs, and drugs, based on selected articles, is shown in Table 2. We were interested to see if the characteristics of reporters (HPs vs. patients) were evaluated by the authors of selected articles as similar or different.

\begin{tabular}{|c|c|c|c|c|c|c|c|c|c|c|c|c|c|c|c|c|c|}
\hline \multirow{2}{*}{$\begin{array}{l}\text { Group of ADR reports from patients } \\
\text { Responses based on questionnaires } \\
\text { send from national regulatory authorities }\end{array}$} & \multicolumn{6}{|c|}{$\begin{array}{l}\text { I } \\
\begin{array}{c}\text { Direct acceptance for safety signal } \\
\text { generation }\end{array}\end{array}$} & \multicolumn{4}{|c|}{$\begin{array}{l}\text { II } \\
\text { Medically } \\
\text { confirmed }\end{array}$} & \multicolumn{2}{|c|}{$\begin{array}{c}\text { III } \\
\text { Not used for } \\
\text { signal detection }\end{array}$} & \multicolumn{5}{|c|}{$\begin{array}{c}\text { IV } \\
\text { Not accepted }\end{array}$} \\
\hline & 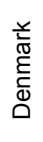 & $\begin{array}{l}\text { 므 } \\
\frac{\mathbb{\sigma}}{\mathbb{D}} \\
\underline{\underline{D}}\end{array}$ & 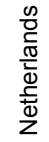 & $\sum_{\substack{0 \\
z}}^{\lambda}$ & 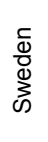 & 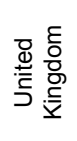 & 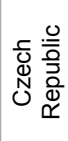 & 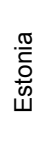 & 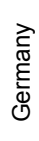 & $\begin{array}{l}\frac{0}{\frac{0}{D}} \\
\frac{0}{\omega}\end{array}$ & 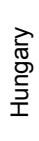 & 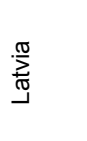 & $\frac{\pi}{\frac{\pi}{2}}$ & 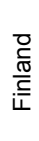 & 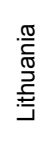 & $\frac{\frac{\pi}{\pi}}{\sum}$ & 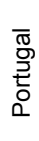 \\
\hline Patients are allowed to send reports & $\mathrm{Y}$ & $\mathrm{Y}$ & $\mathrm{Y}$ & $\mathrm{Y}$ & $\mathrm{Y}$ & $\mathrm{Y}$ & $\mathrm{Y}$ & $\mathrm{Y}$ & $\mathrm{Y}$ & $\mathrm{Y}$ & $\mathrm{Y}$ & $\mathrm{Y}$ & $\mathrm{N}$ & $\mathrm{N}$ & $\mathrm{N}$ & $\mathrm{N}$ & $\mathrm{N}$ \\
\hline Reports used for signal detection & $\mathrm{Y}$ & $\mathrm{Y}$ & $\mathrm{Y}$ & $\mathrm{Y}$ & $\mathrm{Y}$ & $\mathrm{Y}$ & $\mathrm{Y}$ & $\mathrm{Y}$ & $\mathrm{Y}$ & $\mathrm{Y}$ & $\mathrm{N}$ & $\mathrm{N}$ & & & & & \\
\hline Reports are medically confirmed & $\mathrm{N}$ & $\mathrm{N}$ & $\mathrm{N}$ & $\mathrm{N}$ & $\mathrm{N}$ & $\mathrm{N}$ & $\mathrm{Y}$ & $\mathrm{Y}$ & Y & $\mathrm{Y}$ & $\mathrm{N}$ & $\mathrm{N}$ & & & & & \\
\hline
\end{tabular}

European countries were divided into groups of adverse drug reaction reports from patients based on acceptability and use for signal detection. $\mathrm{N}-\mathrm{No}$; $\mathrm{Y}-\mathrm{Yes}$.

Table 1: Processing of adverse drug reaction reports from patients at the national level. 


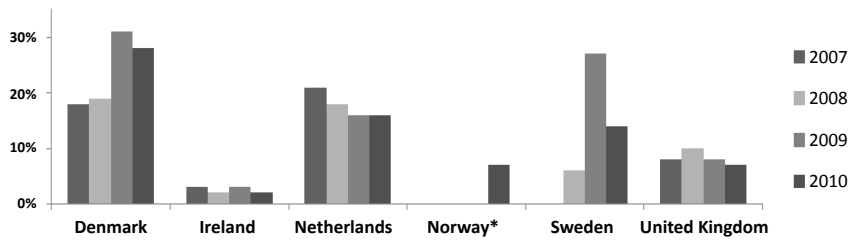

Figure 1: Reporting ratio of adverse drug reaction $(A D R)$ reports from patients to the total number of ADR reports in the six member states of the European Economic Area, where reports from patients are directly accepted and used for signal detection. Data are based on questionnaires received from 17 national regulatory authorities. *Collection of ADR reports from patients began only since 1 Mar 2010

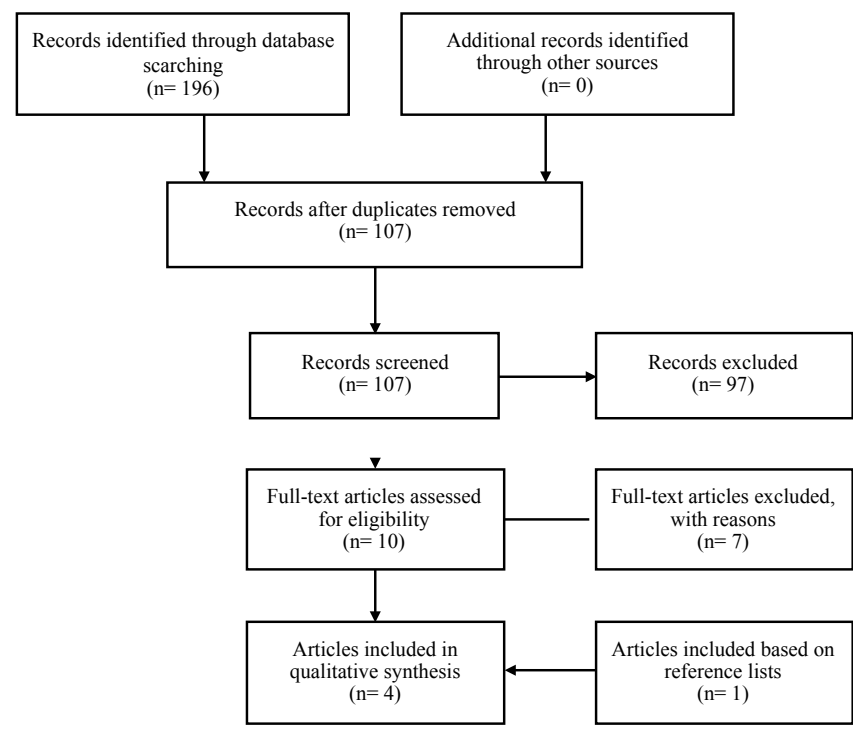

Figure 2: Flow diagram of selection of studies. Structure derived from PRISMA [12,13]

\begin{tabular}{|c|c|c|c|c|c|c|}
\hline \multicolumn{6}{|c|}{$\begin{array}{c}\text { Similarity (1) / } \\
\text { Reporters - Healthcare Professionals }\end{array}$} \\
Author & Country & Age & Gender & $\begin{array}{c}\text { Most Frequently } \\
\text { Reported }\end{array}$ & Seriousness \\
\cline { 4 - 7 } & & & & Drugs & ADRs & \\
\hline de Langen J et al. [15] & NL & 1 & 1 & 1 & 1 & 1 \\
\hline van Hunsel F et al. [16] & NL & -1 & -1 & 0 & -1 & 1 \\
\hline Aagaard L et al. [17] & DK & 0 & 0 & -1 & -1 & -1 \\
\hline McLernon DJ et al. [18] & UK & 1 & 1 & -1 & -1 & -1 \\
\hline
\end{tabular}

Similarity / disparity is marked by 1 - similar; -1 - different; 0 - not evaluated; ADRs - adverse drug reactions; DK - Denmark; NL - the Netherlands; UK - the United Kingdom. Varying results were detected among respective articles (columns) and characteristics (lines) of each article except for that from de Langen et al. [15], where all characteristics of the patients as direct reporters were similar to those of healthcare professionals.

Table 2: Article outcomes - similarities and disparities.

The Netherlands: In 2008, de Langen et al. analyzed reports from HPs (consisting of general practitioners, specialist doctors and pharmacists) and patients received by the Netherlands Pharmacovigilance Centre between April 2004 and April 2007. Patients submitted 2522 reports concerning 5401 ADRs. HPs submitted 10635 reports concerning 16 722 ADRs. This means that each patient report contained, on average, 2.1 ADRs, whereas HPs' reports had 1.6 ADRs. Regarding the reports, the mean age of patients ( 48 years) was similar to HPs ( 49 years). Also,
$63 \%$ of the female patients were comparable to $61 \%$ from HPs. Statins were the most frequently reported drugs for patients and HPs. Moreover, the top five drugs showed great similarity. Also, similarity between reports from patients and HPs concerning the System Organ Class was observed. Finally, the seriousness of the reports was not significantly different from patients (19.5\%) and HPs (21\%) [15]. Comparison of the 3-year period resulted in the acknowledgement of no differences in terms of age, gender, the most frequently reported ADRs and drugs, and the percentage of serious ADRs in general between patients and HPs.

Another study was performed by van Hunsel et al. in the Netherlands. Patients submitted 265 reports, concerning 780 ADRs, about statins to the Dutch safety database from March 2007 to August 2007. HPs submitted 111 reports involving 172 ADRs about statins in the same period. This means that each patient report contained, on average, 3.0 ADRs per patient report, in comparison to 1.5 ADRs per HP report. Patients who reported ADRs were younger ( 57.3 years) than HPs (61.9 years). Of the patient reports, $64 \%$ were male in comparison to $52 \%$ of HPs. An overlap exists in the top 10 of the most frequently reported ADRs, however patients reported more in musculoskeletal disorders and psychiatric disorders. No substantial differences were observed in the percentage of reported seriousness among patients (15.1\%) and HPs (11.7\%); which was the only similar characteristic of ADR reports submitted by patients and HPs [16].

Denmark: Aagaard et al. analyzed 6319 ADR reports related to 15531 ADRs extracted from the Danish safety database for the period 2004 to 2006. Patients submitted 544 ADR reports corresponding to 1700 individual ADRs. The rest of 5775 ADR reports, which were submitted by physicians, pharmacists, other HPs and lawyers included 13831 ADRs. Each patient report contained, on average, 3.1 ADRs, whereas other source of reports had 2.4 ADRs. Age and gender of the reports was not analyzed in the article. There was a significant difference in the distribution of ADRs by type of reporter and System Organ Class or type of drug. Additionally, $46 \%$ of the ADRs reported by patients were classified as serious in comparison to $76 \%$ from physicians, pharmacists and other HPs. Comparison of the 2-year period resulted in the acknowledgement of differences in terms the most frequently reported ADRs and drugs, and the percentage of serious ADRs in general between patients and other reporters including HPs, other HPs and lawyers [17].

The United Kingdom: A total of 26129 reports from the Medicines and Healthcare products Regulatory Agency were analyzed by McLernon et al. which were received from October 2005 to September 2007. Of these, 5180 were patient reports and 20949 were HPs. Patients reported 20358 ADRs (3.9 ADRs per report) whereas HPs reported 44429 ADRs (2.1 ADRs per report). The median age of reporters was similar for patients (54 years) and HPs (53 years). Also gender representation was similar to patient reports (62.7\% females) and HPs (57\%). Reporting forms for patients were slightly different as the patients were not asked the seriousness of the ADR but only the severity of the reaction. Seriousness is characterized by consequences of ADR (e.g. death, hospitalization). Assessment of severity is largely subjective. Reactions can be described as mild, moderate, severe, or lethal in the patient report. Therefore MHRA evaluated the seriousness of the reaction based directly on the report by the patient. $55.5 \%$ of HPs considered reports as serious in comparison to $44.8 \%$ patients declaration that suspected ADR was bad enough to affect everyday activities. More patient reports mentioned a nervous system problem (41.5\%), however the most common System Organ Class for HPs was 
skin and subcutaneous tissue (23.2\%). Also differences were detected in terms of suspected drugs. A comparison of patients' and HPs' ADRs resulted in the acknowledgement of differences in the seriousness and the most frequently reported drugs and ADRs [18].

\section{Strengths and Limitations of the Study}

This study covers the European countries to outline the comparison of position ADR reports submitted by patients. The major strength was the collaboration with the 17 national regulatory authorities, which enabled to us to obtain a general overview of position and processing ADRs in 2011. However, questions concerned to requirements for validation ADR reports or characteristic of method of reporting ADR reactions would require more in-depth analysis to better understand the real impact of patients ADR reports in the national safety database. It would be advantageous to understand the formal validation process of reports and the number of excluded reports. To compare characteristics of reports submitted by patients and HPs was reviewed a literature related to ADR reports in countries, where ADR reports submitted by patients were already accepted.

\section{Risk of Bias}

Selection bias: The selection bias of the literature search could be considered as very low. It was not a coincidence that the articles detected by the search used data from the national databases of the Netherlands, Denmark and the United Kingdom, as their national pharmacovigilance systems are of very high level compared with the other European countries.

Selective reporting bias: Selective reporting bias in the three out of four articles was assessed as having a low risk, as there were included all ADR reports included in the national safety database. In the Dutch study performed by van Hunsel et al. were ADR reports investigated selectively related to statin use after media attention. Therefore, the reporting might be influenced by information presented in media. Patients submitted 265 reports concerning 780 ADRs. HPs submitted 111 reports involving 172 ADRs about statins in the same period. This means that each patient report contained, on average, three ADRs, whereas HPs' reports had 1.5 ADRs. The total number of ADR reports in this period was 833 for patients and 1609 for health professionals [16].

Outcome data bias: ADR reports investigated in the selected articles were extracted from national safety databases. These reports were previously processed and filtered for the purposes of each particular study. It would be beneficial to work with all initial ADR reports submitted by HPs and patients that were not yet processed and included in the national safety databases. For example, in the study by Aagaard et al. [17] was mentioned as limitation of the study that there were investigated consumer reports from Danish ADR database and not the original reports. Therefore validity of ADR reports could not be evaluated [17]. Also, one of the limitations in the study performed by McLernon et al. was incompleteness of the certain the fields in the patient reports so there was a large proportion of missing data [18].

\section{Discussion}

The national pharmacovigilance systems in the 17 European countries, whose responses to questionnaires were received in this study, were established in the years from 1963 to 2004. This broad time span indicates the diversity of the respective systems. Based on data from Bahri et al. ADR reports from HPs were mandatory in most of the countries ( 11 vs. 5 ; data for Norway were not available). ADRs were generally collected only by national centers; in six cases, regional centers and/or major hospitals were also used to support the collection of ADR reports [19]. Pharmacovigilance activities and outcomes are highly dependent on the cultural traditions and attitudes of doctors. In the comparison of any pharmacovigilance data across European countries, we should always keep in mind the variable history of national pharmacovigilance systems and their development, despite the coordination of pharmacovigilance procedures and applications by the EMA since 1993.

\section{Processing ADR reports from patients}

This is the first summary review to present the different attitudes of national regulatory authorities to ADR reports from patients. The majority of national regulatory authorities declared the acceptance of ADR reports directly from patients. Nevertheless, variations existed in the further processing of these reports.

Regulatory authorities in Hungary and Latvia declared that they did not use ADR reports from patients for signal detection by 2011 . Reports collected from patients cannot therefore influence any safety issue related to the use of the drugs in these countries. The reason for the collection of ADRs directly from patients could be to support and cooperate with patients regarding pharmacovigilance activities. Nevertheless, in case of acceptance ADR reports submitted by patients, attention should be focused to the utilization of these reports for signal detection.

In several countries, ADR reports are medically confirmed prior to their inclusion in a safety database, as stated by national regulatory authorities in the Czech Republic, Estonia, Germany and Slovenia. Therefore, the number of patient reports is reduced by the unknown quantity of those excluded during the assessment procedure. Additionally, a more detailed exploration of the effectiveness and administrative burden of medical confirmation should be undertaken.

In several countries (Denmark, the Netherlands and the United Kingdom), ADR reports from patients are accepted without any medical confirmation and are used in safety databases for signal detection. The only control that is possibly provided by regulatory authorities is a formal evaluation (for completeness of the report); however, this cannot be stated for certain, as it was not part of the questionnaire analysis.

As can be seen, there is a disparity in the processing of ADR reports from patients. Different attitudes about the collection and use of ADR reports from patients pointed to the problematic position of direct reporting by patients across European countries. The question how this will be changed with the implementation of new European legislation since July 2012 directing that ADR reports from patients should always be collected $[3,4]$.

\section{Reporting ratio}

Various reporting ratios of ADR reports from patients to the total number of reported ADRs could be observed among different countries. Moreover, various reporting ratios were observed throughout the years in some of the countries. It should be kept in mind that collecting ADR reports from patients has only recently started and its position is currently being formulated in established national pharmacovigilance systems. General factors like legal framework conditions, technical resources, collaboration with stakeholders, and general quality management may influence the submission ADR reports directly by patients [20]. 
Citation: Srba J, Vlcek J (2014) Position and Processing of Adverse Drug Reactions Directly Submitted by Patients to National Regulatory Authorities in Europe. J Pharmacovigilance 2: 122. doi:10.4172/2329-6887.1000122

Page 5 of 5

\section{Comparison of ADR reports}

Varying outcomes were detected across the analyzed articles that compared ADR reports from HPs and patients in terms of age and gender of reporters, most frequently in reported ADRs and drugs and in the seriousness of reports. A systematic review of comparative studies by Inch et al. revealed both differences and similarities between reporter types [21]. As a limitation of our study, it should be acknowledged that the studies analyzed did not clearly describe the assessment process of ADR reports when they are first received by national regulatory authorities. It can be assumed that all reports were probably already controlled on the basis of data quality, and that some of them could also be medically confirmed prior to incorporation into the safety database. To enhance the validity of the data that were compared, studies should always describe the similarities and differences in the processing of reports after ADR collection.

\section{Conclusions}

Spontaneous ADR reporting by patients has become a valuable pharmacovigilance tool and has already contributed to safety signal generation $[7,22]$. At this moment, ADR reports directly submitted by patients are becoming more or less an integral part of national pharmacovigilance systems as an additional source of reports for the generation of safety signals. The comparison of ADR report processing from patients and from HPs revealed differences in terms of acceptance of ADR reports from patients, their medical confirmation, and their inclusion in the safety database, which is necessary for signal generation. Moreover, various outcomes were observed across studies that compared characteristics of reporters, drugs, and ADRs between HPs and patients.

This study revealed the need for analysis of the effective use of ADR reports from patients in the national pharmacovigilance systems, particularly in the processing of ADR reports from patients from the time they are collected by national regulatory authorities.

\section{Acknowledgement}

This work was supported by scientific grant SVV 267005.

\section{Conflicts of Interest}

The authors have no conflicts of interest.

\section{Ethical Statement}

Statement of ethical approval was not required as the research did not involve data concerned to human participants. The project only used official statistical data.

\section{References}

1. Mann RD, Andrews EB (2007) Pharmacovigilance. (2nd edn), John Wiley and Sons, Chichester, England.

2. VOLUME 9A of The Rules Governing Medicinal Products in the European Union, Guidelines on Pharmacovigilance for Medicinal Products for Human Use, September 2008.

3. Regulation (EU) No 1235/2010 of the European Parliament and of the council of 15 December 2010, amending, as regards pharmacovigilance of medicinal products for human use, Regulation (EC) No 726/2004 laying down Community procedures for the authorisation and supervision of medicinal products for human and veterinary use and establishing a European Medicines Agency, and Regulation (EC) No 1394/2007 on advanced therapy medicinal products.

4. Directive 2010/84/EU of the European Parliament and of the council of 15 December 2010 amending, as regards pharmacovigilance, Directive 2001/83/ EC on the Community code relating to medicinal products for human use.

5. van Grootheest K, de Graaf L, de Jong-van den Berg LT (2003) Consume adverse drug reaction reporting: a new step in pharmacovigilance? Drug Saf 26: $211-217$
6. Vincent CA, Coulter A (2002) Patient safety: what about the patient? QualSaf Health Care 11: 76-80.

7. van Hunsel F, Härmark L, Pal S, Olsson S, van Grootheest K (2012) Experiences with adverse drug reaction reporting by patients: an 11-country survey. Drug Saf 35: 45-60.

8. Herxheimer A, Crombag R, Alves TL(2010) Direct patient reporting of adverse drug reactions: a fifteen-country survey and literature review.

9. van den Bemt PM, Egberts AC, Lenderink AW, Verzijl JM, Simons KA, et al (1999) Adverse drug events in hospitalized patients. A comparison of doctors, nurses and patients as sources of reports. Eur J ClinPharmacol 55: 155-158.

10. Jarernsiripornkul N, Krska J, Capps PA, Richards RM, Lee A (2002) Patient reporting of potential adverse drug reactions: a methodological study. $\mathrm{Br} \mathrm{J}$ Clin Pharmacol 53: 318-325.

11. Lopez-Gonzalez E, Herdeiro MT, Figueiras A (2009) Determinants of underreporting of adverse drug reactions: a systematic review. Drug Saf 32: 19-31.

12. Avery AJ, Anderson C, Bond CM, Fortnum H, Gifford A, et al. (2011) Evaluation of patient reporting of adverse drug reactions to the UK 'Yellow Card Scheme': literature review, descriptive and qualitative analyses, and questionnaire surveys. Health Technol Assess 15: 1-234.

13. The PRISMA statement.

14. Moher D, Liberati A, Tetzlaff J, Altman DG; PRISMA Group (2009) Reprint-preferred reporting items for systematic reviews and meta-analyses: the PRISMA statement. Phys Ther 89: 873-880.

15. de Langen J, van Hunsel F, Passier A, de Jong-van den Berg L, van Grootheest $\mathrm{K}(2008)$ Adverse drug reaction reporting by patients in the Netherlands: three years of experience. Drug Saf 31: 515-524.

16. van Hunsel F, Passier A, van Grootheest K (2009) Comparing patients' and healthcare professionals' ADR reports after media attention: the broadcast of a Dutch television programme about the benefits and risks of statins as an example. Br J Clin Pharmacol 67: 558-564.

17. Aagaard L, Nielsen LH, Hansen EH (2009) Consumer reporting of adverse drug reactions: a retrospective analysis of the Danish adverse drug reaction database from 2004 to 2006. Drug Saf 32: 1067-1074.

18. McLernon DJ, Bond CM, Hannaford PC, Watson MC, Lee AJ, et al. (2010) Adverse drug reaction reporting in the UK: a retrospective observational comparison of yellow card reports submitted by patients and healthcare professionals. Drug Saf 33: 775-788.

19. Bahri P, Tsintis P, Waller PC (2007) Regulatory Pharmacovigilance in the EU. Pharmacovigilance (2ndedn), John Wiley and Sons Ltd, Chichester, United Kingdom.

20. Bührlen B, Reiß T, Beckmann C, Gassner UM, Gleiter CH (2006) Assessment of the European Community System of Pharmacovigilance. Final Report-Final Version, European Commission.

21. Inch J, Watson MC, Anakwe-Umeh S (2012) Patient versus healthcare professional spontaneous adverse drug reaction reporting: a systematic review. Drug Saf 35: 807-818.

22. Härmark L, van Grootheest AC (2008) Pharmacovigilance: methods, recent developments and future perspectives. Eur J Clin Pharmacol 64: 743-752. 